\title{
Covoitureurs des villes, covoitureurs des champs. Une analyse spatialisée du covoiturage en France
}

Eléonore Pigalle \& Anne Aguiléra 


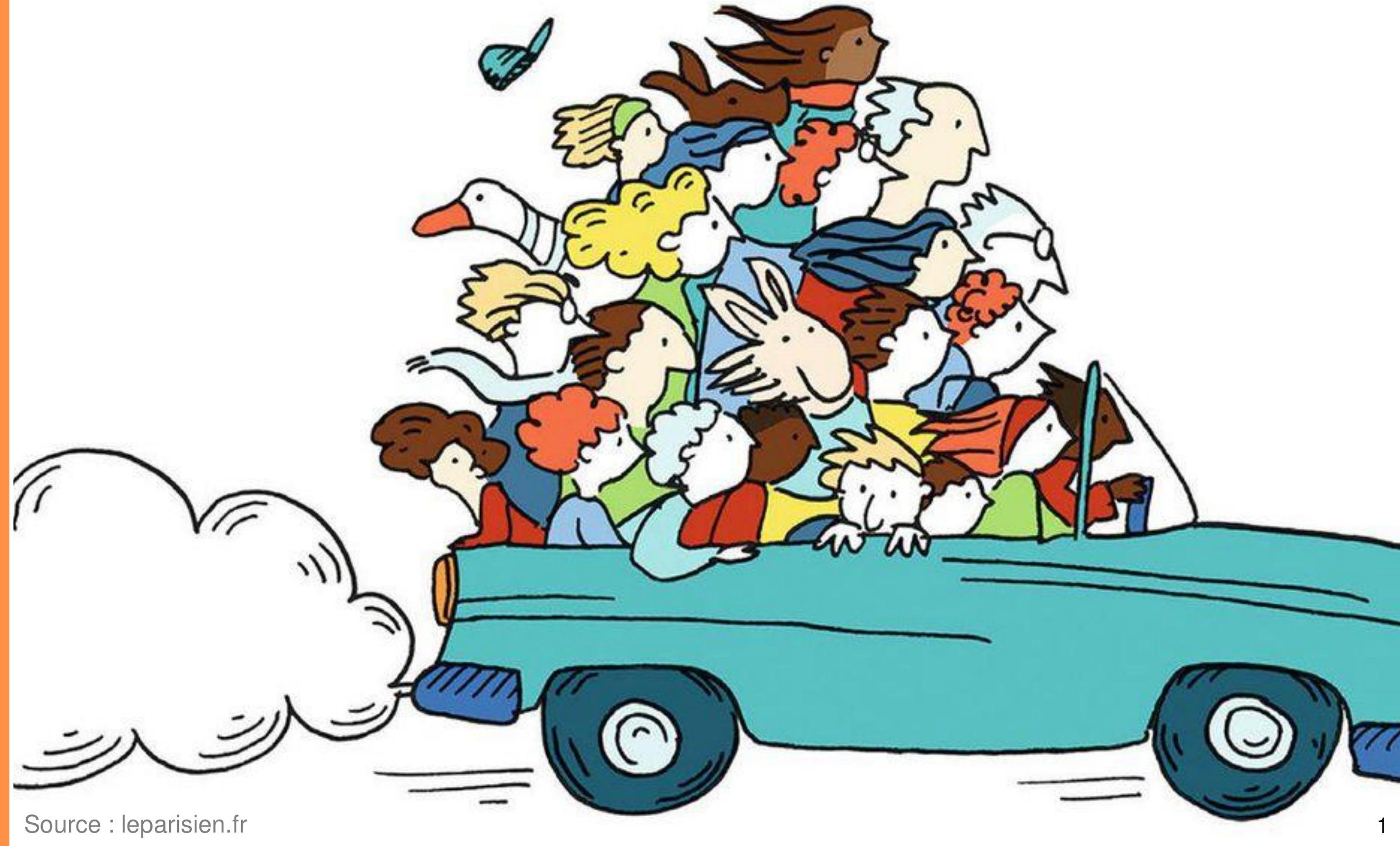




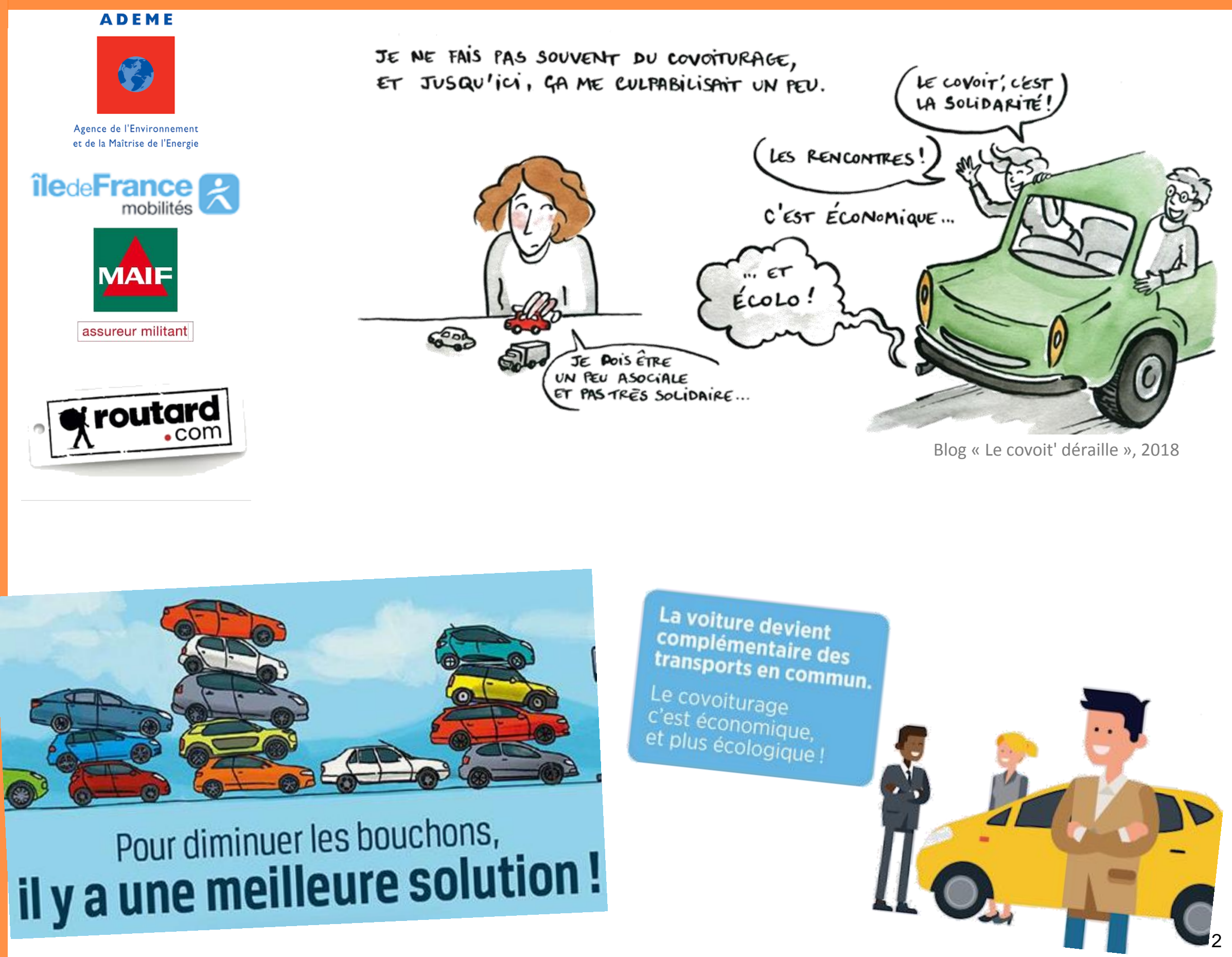




\section{Le Gouvernement se mobilise pour faire du covoiturage:}

une évidence au quotidien
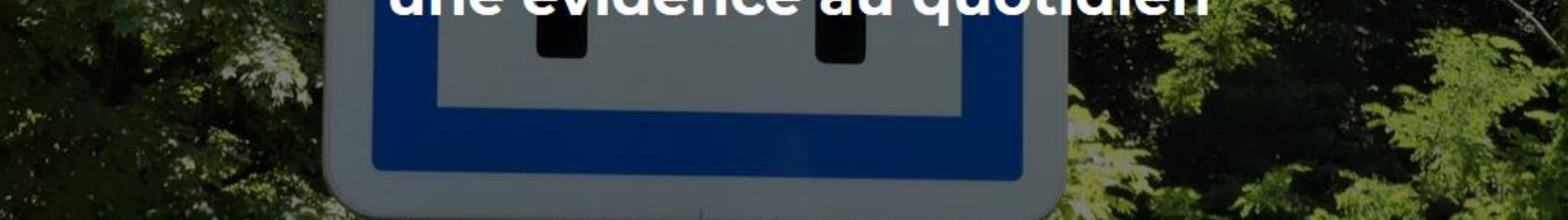

\section{Techni Cités}

Cent lignes de covoiturage à déployer dans les territoires peu denses Publié le 17/12/2019 Par Thomas Blosseville • Club : Club Techni.Cités

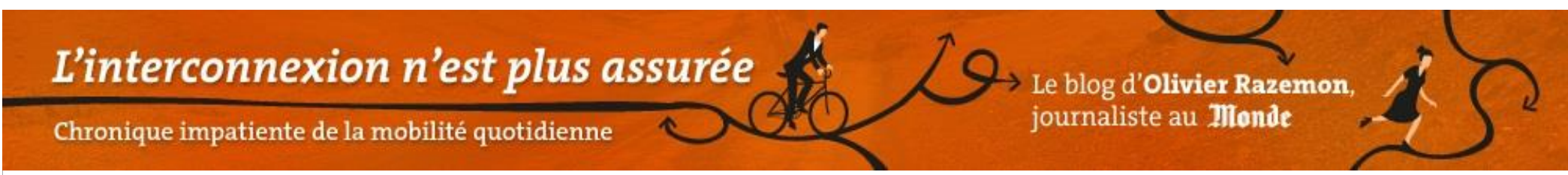

«Territoires peu denses »: ces petits détails qui pourraient faciliter les déplacements

Publié le 29 septembre 2019 


\section{ProblématiQue}

\section{Peu denses}

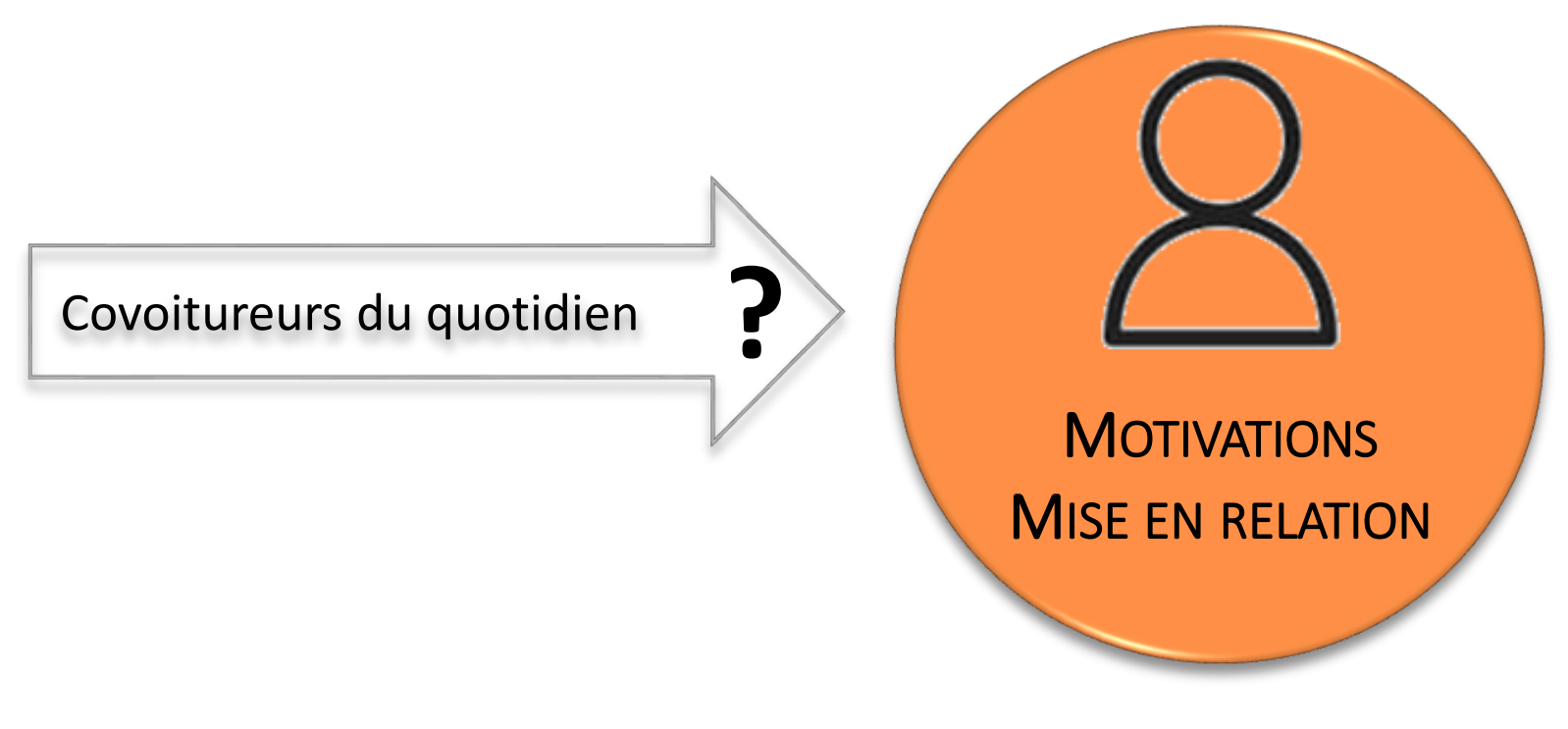

\section{DENSES}

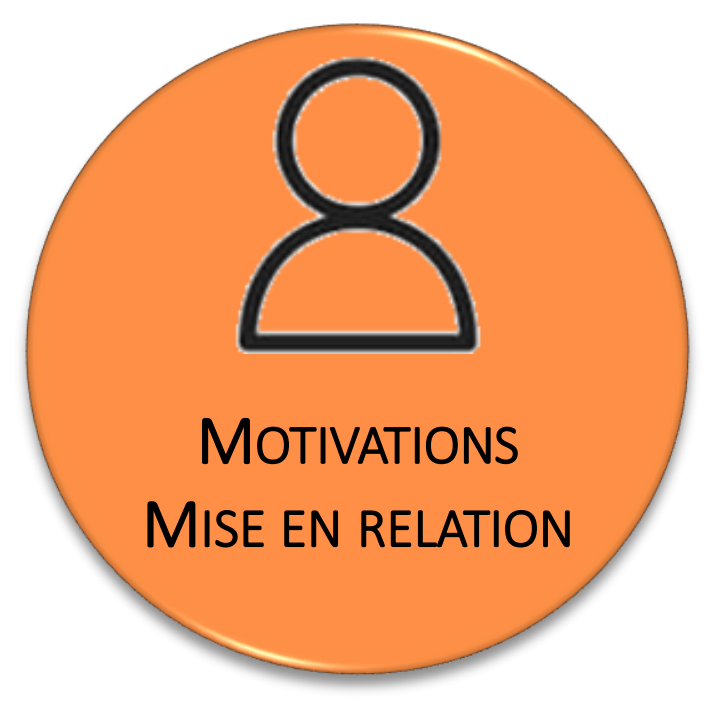




\section{MÉTHODE}

$\square$ Enquête par questionnaire ( $n=2000), 2016$ - GIS Marsouin
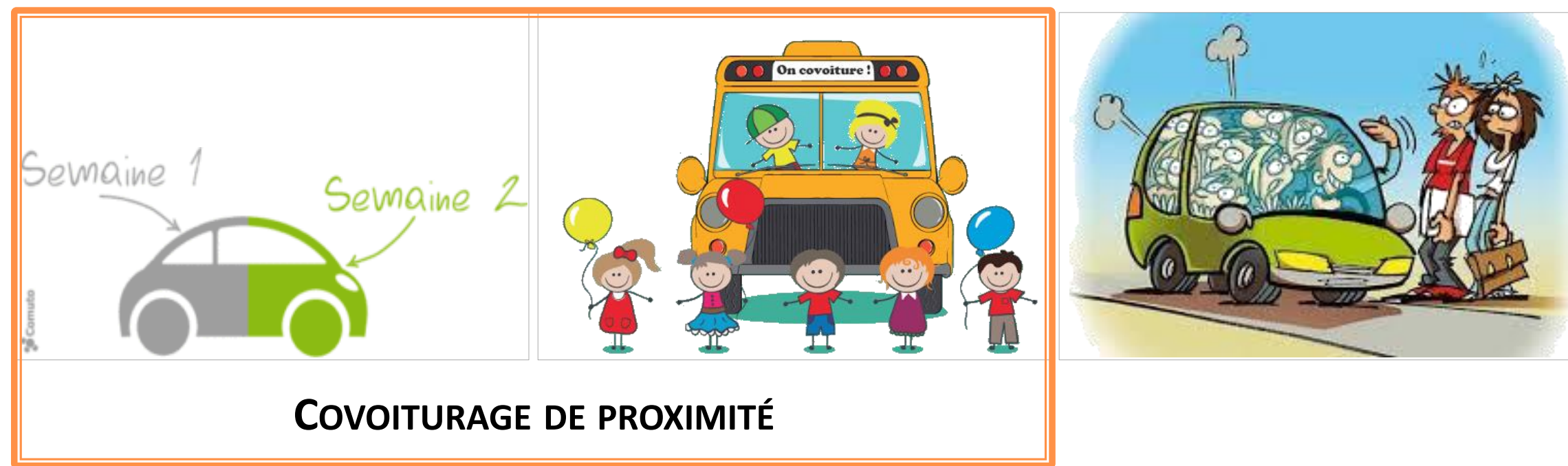

$\square$ Limites : conducteurs/passagers 


\section{EN PRATIQUE}

Au total : $23 \%$ de covoitureurs de proximité

\begin{tabular}{|c|c|}
\hline & COVOITUREURS DE PROXIMITÉ \\
\hline $\begin{array}{r}\text { COMMUNES PEU DENSES } \\
\qquad(n=400)\end{array}$ & $25 \%$ \\
\hline $\begin{array}{r}\text { COMMUNES DENSES } \\
(n=583)\end{array}$ & $21 \%$ \\
\hline
\end{tabular}




\section{DÉTERMINANTS DE LA PRATIQUE DU COVOITURAGE DE PROXIMITÉ}

\section{DENSITÉ}

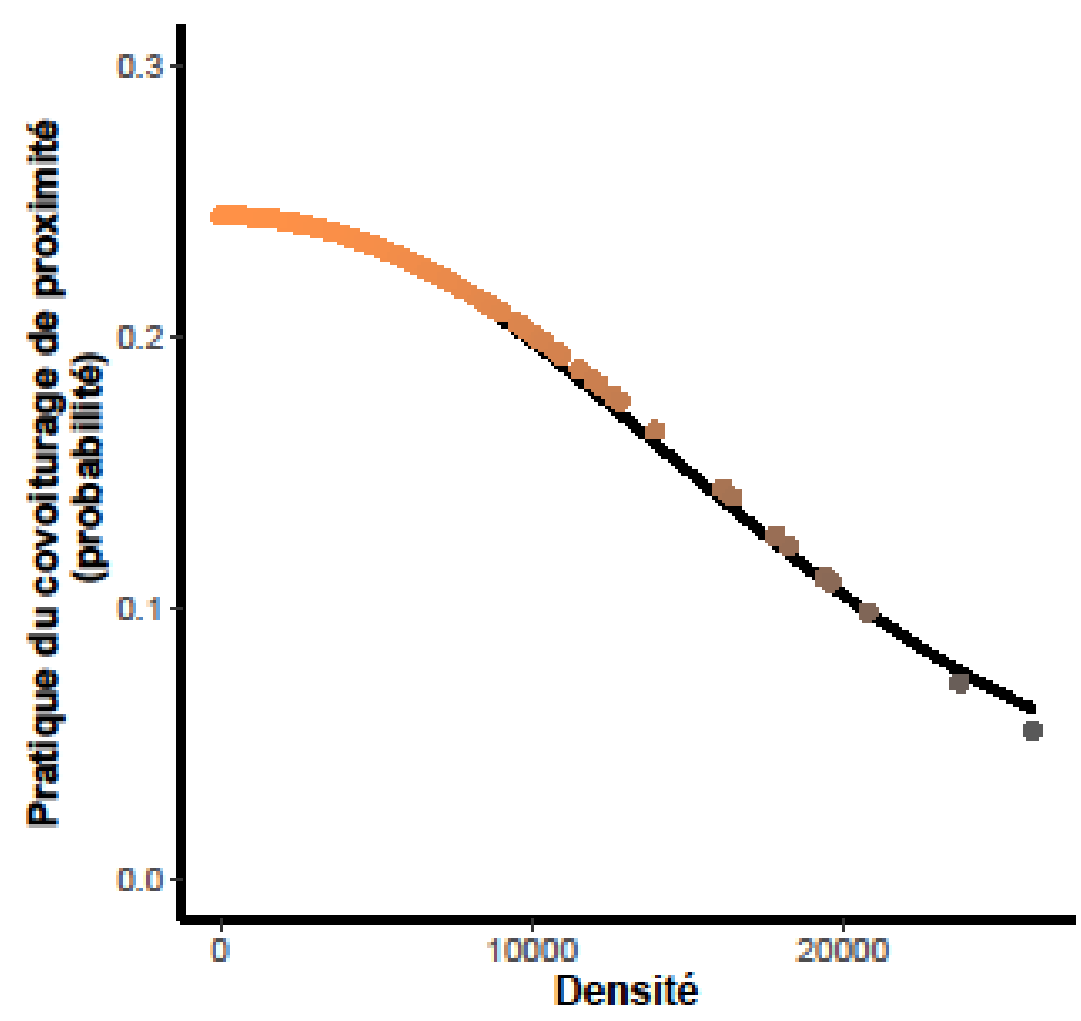

(Olsson et al., 2019) 


\section{DÉTERMINANTS DE LA PRATIQUE DU COVOITURAGE DE PROXIMITÉ}

\section{DENSITÉ}

\section{AUTOMOBILE}

NOMBRE

HABITUDE MODALE
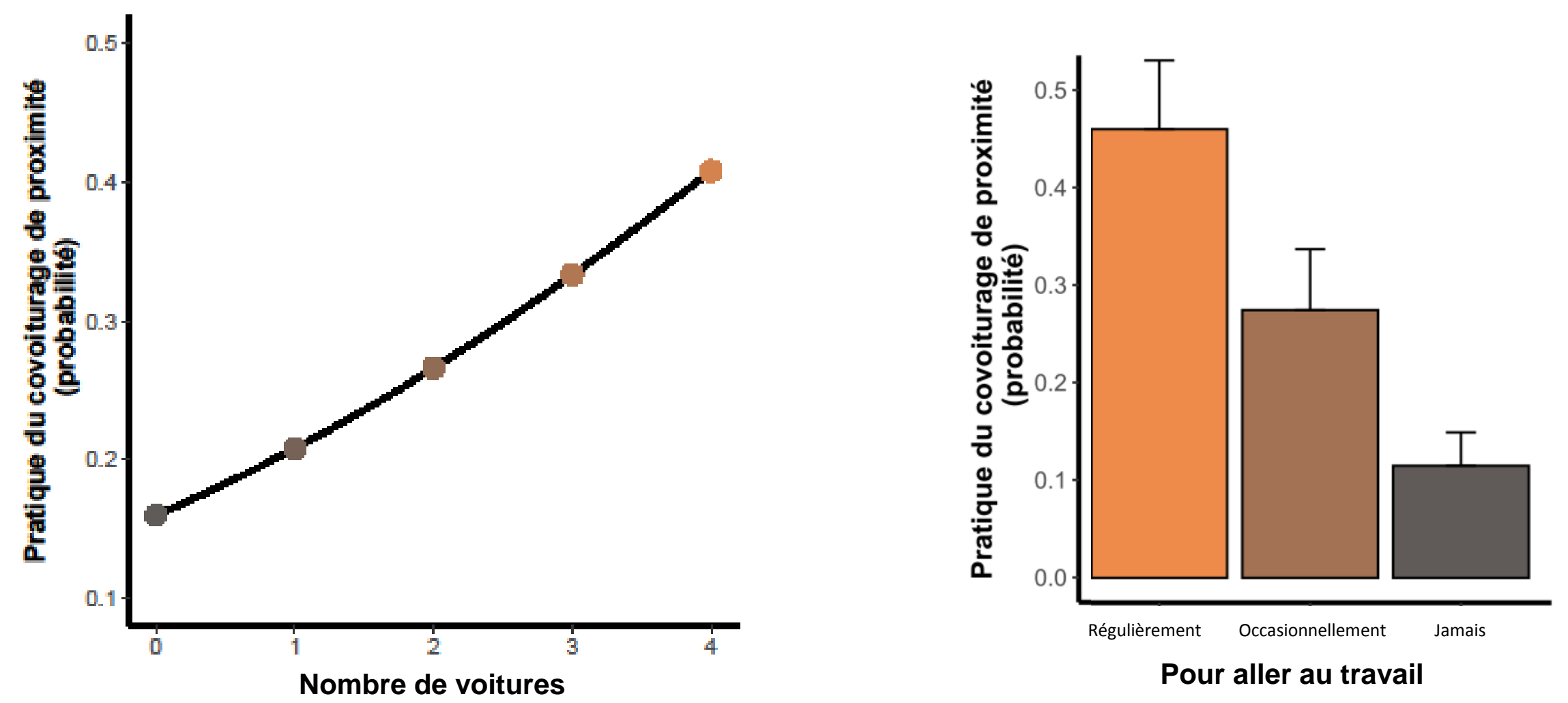


\section{DÉTERMINANTS DE LA PRATIQUE DU COVOITURAGE DE PROXIMITÉ}

\section{DENSITÉ}

\section{AUTOMOBILE}

NOMBRE

HABITUDE MODALE

\section{REPRÉSENTATIONS}

AUTOMOBILE

TRANSPORT EN COMMUN
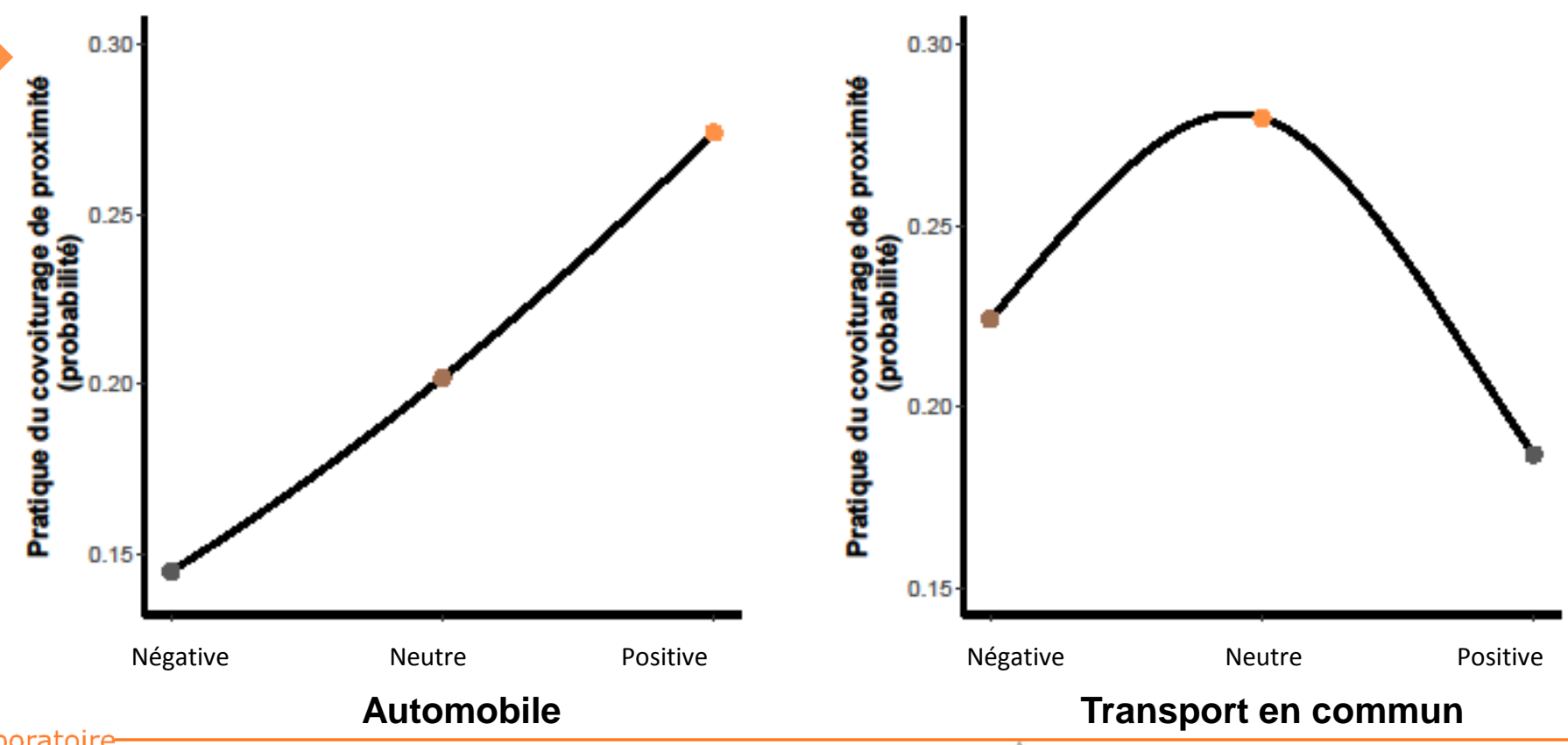


\section{DÉTERMINANTS DE LA PRATIQUE DU COVOITURAGE DE PROXIMITÉ}
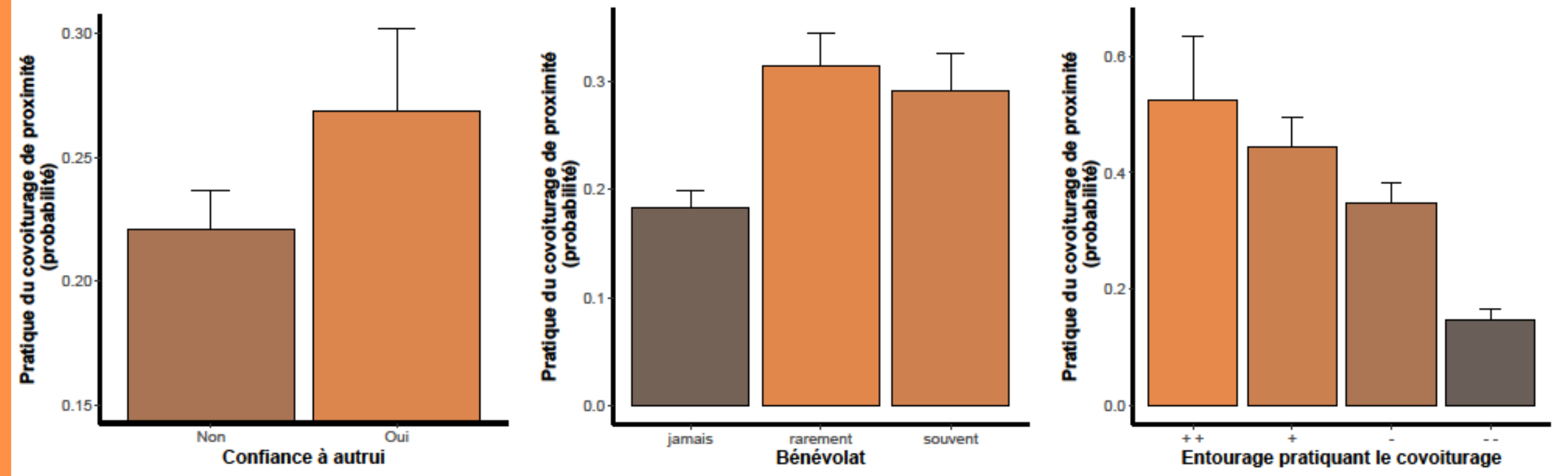

\section{RELATIONS}

CONFIANCE BÉNÉVOLAT COVOITUREURS 


\section{DÉTERMINANTS DE LA PRATIQUE}

\section{DENSITÉ}

\section{AUTOMOBILE}

NOMBRE

HABITUDE MODALE

\section{REPRÉSENTATIONS}

AUTOMOBILE

TRANSPORT EN COMMUN

\section{RELATIONS}

CONFIANCE

BÉNÉVOLAT

COVOITUREURS

\section{IDÉES}

\section{IDES}


... de partager des émotions authentiques et enrichissantes

... c'est plus pratique, tout simplement

... je crois qu'on pourrait changer le monde

... de reprendre en main le contrôle de sa propre vie

... de remettre plus de sens dans la vie en société

... d'être indépendants des modèles de consommation imposés par les grands groupes industriels ou financiers

... un moyen de participer à la préservation de notre planète

... de rencontrer des personnes intéressantes

... de retrouver de l'autonomie dans notre façon de consommer

... de favoriser des échanges entre humains et pas seulement des échanges de biens

L'existence même de la consommation collaborative prouve que l'on est capable d'être plus autonome vis-à-vis de grands systèmes

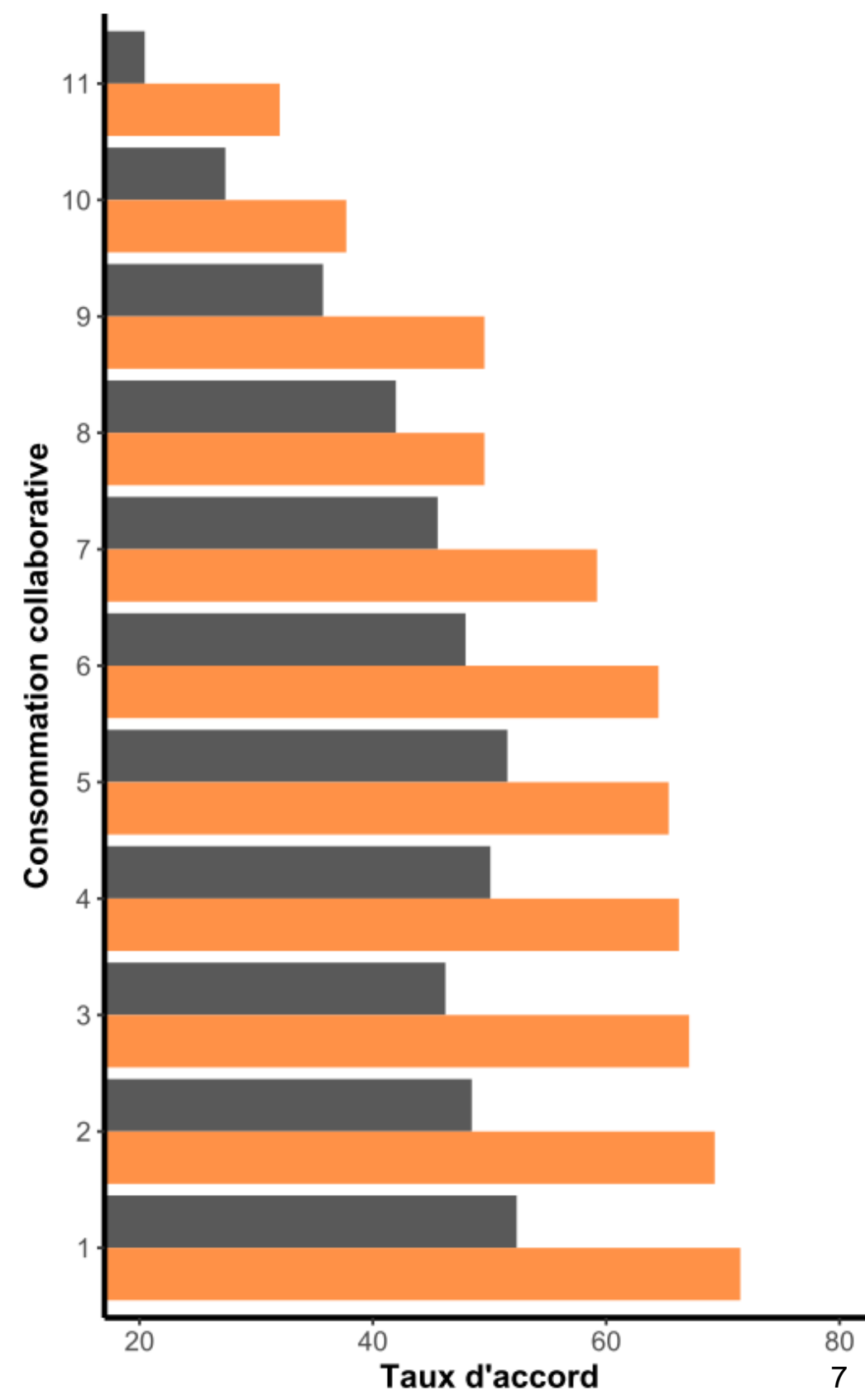




\section{DENSES}

\section{PROFIL SOCIO-ÉCONOMIQUE}

DIPLÔME

Sans diplôme, Brevet des collèges,

BAC, +1 et +2

$\mathrm{BAC}+3$ et plus

REVENU DU MÉNAGE (PERÇU)

Je m'en sors

Je trouve la vie difficile

\section{AUTOMOBILE}

POSSESSION

REPRÉSENTATIONS

Plus d'avantages

Plus d'inconvénients

TRANSPORTS EN COMMUN

QUALITÉ DE LA DESSERTE

REPRÉSENTATIONS

\section{Covolturage}

PARTAGE DES FRAIS

Très rarement

Régulièrement

Mise EN RELATION

Arrangements avec des connaissances 


\section{POUR CONCLURE}

Covoiturage de proximité

- Être bénévole, faire confiance aux autres, connaître des covoitureurs

- Adhésion à des valeurs alternatives de consommation et de société

- Pratique et représentation positive de l'automobile

- Densité faible

- Réseaux de proximité

- Faible partage de frais 


\section{Merci de votre attention}

